\section{Risk management in clinical practice. Part 1. Introduction}

\author{
L. D'Cruz'
}

IN BRIEF
- Complaints are a recognised feature
of dental practice but they arise only
when precipitating factors occur with
predisposing factors.
- Good communication is the key to
reducing patient complaints.
Record keeping is an essential skill and
the ten key features of good record
keeping are described.
Risk management concepts are explored
with emphasis on learning from mistakes.

This paper introduces a number of concepts that will recur throughout the series. It examines firstly the rationale behind what triggers patients' complaints and explores the concepts of precipitating and predisposing factors. If patients are dissatisfied they may seek legal redress and clinical negligence is explained briefly with an explanation particularly of breach of duty and causation. Record keeping is an integral part of delivering high quality care and ten essential requirements are listed. Where patients request it or treatment is more complex than the practitioner can manage, a referral should be considered. An outline of risk management principles are given with emphasis on learning from mistakes.

We currently practise at a time when government policy, regulation of the profession and patients' expectations make quite significant demands on dental teams. Patients expect to be offered choice for some if not all the treatments we provide and this expectation is enshrined in the statute law of consent, in ethical guidance

\section{RISK MANAGEMENT IN CLINICAL PRACTICE \\ 1. Introduction \\ 2. Getting to 'yes' - the matter of consent \\ 3. Crowns and bridges \\ 4. Endodontics \\ 5. Ethical considerations for dental enhancement procedures \\ 6a. Identifying and avoiding medico-lega risks in complete denture prosthetics \\ 6b. Identifying and avoiding medico-legal risks in removable dentures \\ 7. Dento-legal aspects of orthodontic practice \\ 8. Temporomandibular disorders \\ 9. Dental implants \\ 10. Periodontology \\ 11. Oral surgery}

'Dento-legal Advisor, Dental Protection Ltd/General Dental Practitioner, 6 The Broadway, Woodford Green, Essex, IG8 OHL

Correspondence to: Len D'Cruz

Email:lendcruz@btinternet.com

\section{Refereed Pape}

Accepted 20 October 2009

DOI: 10.1038/sj.bdj.2010.579

${ }^{\oplus}$ British Dental Journal 2010; 209: 19-23 from regulatory bodies and NHS policy.

In this world of consumerism and high expectations, patients are sometimes dissatisfied about treatment, which results in complaints. Complaints in dentistry can be categorised into those situations where something has gone badly wrong (fractured maxillary tuberosity during extraction or a foreign body inhaled during treatment) and others where the dentist or team have failed to satisfy the expectations of their patients (a crown drops out within a week of fitting or the patient has problems with dentures). A degree of motivation is required for any patient to register dissatisfaction. The greater the gap between expectations and outcome, the higher the level of motivation to complain.

\section{PRECIPITATING AND PREDISPOSING FACTORS}

Patients can and do complain about the dental care they receive. A complaint may be related specifically to the clinical treatment received or it may relate to administrative issues or interpersonal communications with staff. What this demonstrates is that complaints occur when precipitating and predisposing factors occur simultaneously. ${ }^{1}$

Precipitating factors are those that actually give rise to the complaint such as an adverse outcome, providing incorrect care and system errors and mistakes. This could range from the laboratory work not being delivered in time for the patient's appointment to an anaphylactic reaction following the administration of a local anaesthetic.

A predisposing factor on its own does not result in a complaint but increases the chance of it occurring, for example rudeness, delays, lack of attention, apathy or poor communication.

It is when the precipitating factors are overlaid with the predisposing factors that the patients can direct the blame at a series of individuals and a complaint crystallises. A complaint can be defined as 'an expression of dissatisfaction with the practice's procedures, charges, personnel or quality of service.' ${ }^{2}$

For the reasons outlined above it is clear that not every dissatisfied patient complains. Many simply decide not to return to the practice and some of these will tell others. Nine out of ten dissatisfied patients do not usually give the practice a chance to put things right and leave without raising their concerns. ${ }^{3}$ This is a double-edged sword, as it might save the practice from a complaint and the attendant problems but it also denies the opportunity of preventing a similar problem recurring.

If an individual is personable and pleasant and a good communicator there is reduced risk of receiving a complaint or being sued. This was highlighted in a study by Di Matteo ${ }^{4}$ who found that communication skills, particularly nonverbal skills, had a significant influence 
on a patient's satisfaction level towards outcomes of treatment. This fact was demonstrable regardless of the quality of care provided. ${ }^{5}$

The patient carries with them a unique set of expectations and the challenge is to first identify those expectations and then meet them. Patients rarely come to a practice as a 'blank canvas'. Many of then arrive with 'excess baggage' accumulated from previous experiences or previous dentists and on occasion a patient's perceptions of dentistry or a particular treatment outcome is based on the experiences of others. ${ }^{6}$ The systems of clinical governance - protocols essentially - ensure that those expectations can be captured, for example from a carefully taken dental history, clinical examinations or lifestyle questionnaire. Those expectations then need to be modified, ie to be made realistic by information provided by the dentist. This is especially so where cosmetic dentistry or removable prosthetics is planned. Once the divide between the patient's expectations and what can actually be achieved is reduced or eradicated, the unmet expectations will be reduced and consequently the cause of many complaints.

There is no doubt that complaints have a significant impact on the clinicians who receive them ${ }^{7}$ and their effect cannot be underestimated. Efforts to reduce complaints or eliminate them as far as possible will benefit all parties.

\section{CLINICAL NEGLIGENCE}

There are many routes for patients seeking redress to their complaints in the UK, one of which is to bring a legal action for negligence against the dentist. For a patient to succeed in any claim for negligence three essential features must be present and proved:

- That a duty of care is owed by the dentist to the patient

- That there was a breach of duty of care in failing to reach the standard of care expected

- That the patient suffered harm/losses as a result (causation) and that the harm was foreseeable.

In most cases the first of these three essentials is satisfied simply when the patient is accepted by the dentist as a patient or examined, and in the context of general practice this will happen when the patient is seen by the dentist normally in a clinical environment. A duty of care then exists whether it is advice or treatment that is then provided.

Much of this series explores the second of these three essential components of negligence. For a claimant to successfully sue their dentist or dental care professional, often with the help of a lawyer and usually an expert dental opinion, they need to demonstrate that the dentist or dental care professional (DCP) did or did not do something that a reasonable dentist or DCP would or would not have done in the same circumstances. This is the Bolam test and it is discussed in detail in the next article on consent. The expectation of what constitutes good practice comes from a general dental practitioner's peers, from guidelines and from an evidence base.

Not taking radiographs when appropriate and failing to warn about a likely risk are two of many examples of what may be required of a dentist in a certain circumstance, the failure of which amounts to a breach of a duty of care.

The third and arguably the most important leg of a patient's negligence claim is that of causation. The claimant has to establish, on the balance of probabilities, that the dentist's negligent act or acts has directly caused the harm or injury for which compensation is being sought. It is at this hurdle that many claimants' cases fail since it is not enough merely to show that the dentist provided substandard care. For example, a poorly completed root canal treatment resulting from a failure to take diagnostic and working length radiographs or any operative measurements may be judged to have been negligently carried out in that a competent practitioner would be expected to carry out these measurements. If, however, the patient has not suffered any damage in terms of pain, loss of function or the requirement to have further treatment, no harm has been caused by the negligent act.

Taking another example, a patient who has periodontal disease and who is not diagnosed as a result of a failing on the dentist's part will need to demonstrate that it was the dentist's failure to diagnose the periodontal disease sufficiently early that caused the loss of attachment or teeth. This has to take into consideration the natural progress of the disease which may have resulted in the losses in any case, regardless of intervention, as well as any contributory negligence on the part of the patient who may have previously been given information and advice about self care but not acted on it. For this defence to be proposed, a dentist's records must demonstrate that this advice was given.

\section{RECORDS}

Clinical dental records are primarily designed to record treatment carried out on a patient and act as a historical record. They are essential to the delivery of dental care, contributing to the processes of diagnosis, treatment planning and the provision of care in an ordered manner. Clinical records are a communication tool allowing a patient's care to be accessed by the treating dentist, the patient themselves and other healthcare workers. ${ }^{8}$

The records assume, however, an important significance when a patient either complains or makes a claim in negligence or something goes wrong. One of the most frustrating aspects of analysing a dentist's notes is discovering their legal position is undermined by the lack of supportive information in the patient's clinical records, even when the treatment provided has been of a high standard.

Record keeping is often considered an afterthought by practitioners, used merely to record treatment items, to note financial or administrative functions and often not much else. The rise of consumerism means patients' expectations of their care is higher and patients are ever more ready to question the care they have been given. This threat of litigation has prompted many dentists to change their note taking and improve the explanations given to patients. ${ }^{9}$

It is often the difference of spending 30 seconds more on making a pertinent contemporaneous entry about a conversation, warning, option or piece of advice that can provide the perfect antidote to the patient's selective memory of the occasion. Often these crucial entries are not there and the pendulum will swing in favour of the patient's recollection of events on the basis that the patient has only one dentist, but the dentist has many patients and his or her memory, in the absence of a written record, will be judged to be less reliable. 
There are ten essential requirements in clinical record keeping: ${ }^{2}$

1. Identification data - these will include name, address, telephone numbers and email addresses. Text messages (SMS [short message service]) are being increasingly used by practices to remind patients of appointments and so mobile phone numbers are useful

2. Medical history - this should be in the form of a written proforma which will cover all aspects of the patient's general health. This should be signed and dated ${ }^{10}$ by the patient and have a space for the treating dentist to date every time the medical history is checked at recalls. Updating the medical history on a computer is equally valid as long as the changes are date stamped. There should be an enquiry about smoking and alcohol consumption and any advice given in relation to reduction or cessation should be noted ${ }^{11}$

3. Dental history - this should cover previous dental experiences, why the patient has come to the particular practice as well as an understanding of risk factors such as diet and oral hygiene measures, and previous dental care. Lifestyle questions about attitudes to dentistry and cosmetic treatment can be covered in a questionnaire

4. Clinical examination - this clinical examination should cover both extraoral as well as intra-oral structures including an oral cancer screening. Both negative and positive findings should be recorded. A baseline charting to include the current status of the teeth and supporting periodontal structures should be undertaken with a record that this has been done. A basic periodontal examination (BPE) or some other equivalent objective measurement should be recorded to assess the periodontal tissues

5. Radiographic examination - any radiographs taken should be justified and the report on any findings should be in the notes. A subjective quality rating is also required in some jurisdictions $^{12}$

6. Diagnosis - unfortunately very few notes record a diagnosis except for the most common, for example pericoronitis. A diagnosis gives a rationale for treatment and should be present even for routine fillings, eg 'recurrent caries - broken filling' or 'irreversible pulpitis'

7. Treatment plan - a list of treatment to be done as well as any referral that needs to be made should be recorded. This allows the proper sequencing of treatment according to appropriate principles of relief of pain first followed by increasingly complex treatment, depending on the patient's response to prevention and other advice

8. Reference to consent - the options available should be discussed and recorded as well as the relevant advantages and disadvantages. The patient's preference for a particular treatment should be recorded along with the reasons for doing so, especially if the dentist is not in complete agreement. More complex or riskier procedures may warrant written consent forms requiring the patient's signature

9. Progress notes - these will form the bulk of the records and should always be dated and a note made of the treating dentist. The nurse's initials are useful also. The treatments undertaken, details of local anaesthetics and any instructions given should be noted. Some warnings may be given as standard and therefore to avoid continually recording them, an advice sheet can be given and a copy retained in the file for future reference. These may be on post-extraction instruction, advice about orthodontic appliances or the care of dentures for example

10. Exit notes - if a patient informs the practice that they are leaving it is useful to record the reasons for the departure. This is particularly the case if they are in the middle of treatment. Many practices send questionnaires to patients who have not visited the practice for some time and these will assist in developing a customer orientated approach to patient care. Some patients may request copies of their notes or radiographs when they leave and they are entitled to copies of them under the Data Protection Act 1998 in England.

It is a common adage that 'if it is not written in the notes, it did not happen' and this relates as much to unusual situations in practice as to routine care. If a patient is given oral hygiene instructions, or advised to use a fluoride mouthwash or to give up smoking, these may be routine everyday interactions but they assume enormous significance at a later date if the patient makes a claim for negligent management of their periodontal disease. Simply recording this advice and their response to it over time will enable a successful defence of the claim to be mounted. Merely noting 'Exam S+P' will not capture what may have been a significant discussion about prevention and the aetiology of gum disease.

\section{ELECTIVE TREATMENT}

When embarking on elective treatment on teeth that are healthy and symptomfree, record taking should be meticulous. Examples of this might include:

- Elective root canal treatment therapy before fixed restorations

- Elective removal of symptom-free third molars or retained roots

- Elective preparation of previously intact (or minimally restored) teeth to receive crowns, bridges, veneers etc

- The decision to remove most or all of a patient's remaining teeth and to provide dentures

- The removal of (usually sound) teeth as part of orthodontic treatment.

The more elective the treatment, the more intrusive/interventionist/irreversible the treatment, the greater the risks of an adverse outcome, or the consequences if this were to happen, then the more critical it becomes to have full and meticulous records available. ${ }^{13}$ This might include study models and clinical photographs in the case of elective cosmetic treatment as well as any correspondence to the patient setting out the discussions that have taken place and confirmation of what is being planned with the attendant costs.

\section{REFERRALS}

A dentist has a duty to refer patients for a second opinion and for further advice 
when it is necessary, or if the patient asks. ${ }^{14}$ This means that a practitioner has a duty to consider, before commencing treatment, whether a particular procedure is something for which he/she has the appropriate training, experience, facilities, skills and competence to carry out safely and to an appropriate standard. It is for the patient to decide whether they wish to have the treatment carried out by a general practitioner, or by a recognised specialist in the relevant field. The latter may cost more, and this is a relevant consideration for the patient as part of the consent process.

For all the reasons above, the patient needs to be provided with sufficient information about the alternatives in order that they are placed in a position to make a properly informed decision before the event. Where the patient is not made aware of these options and the treatment proves unsuccessful, it may well be argued that the patient would have wished to have been referred for treatment by a specialist and this is what they would have chosen if only they had been given this opportunity. ${ }^{15}$

Referrals may be appropriate for complex clinical cases such as periodontal disease, oral surgery, root canal treatments and restorative problems and it is important that practitioners recognise their limitations in managing these treatments. A professional recognises their limitations. These may be based on the realisation that a particular patient's needs cannot be met, either because of the complexity of the treatment or the demands of the patient. It is neither a sign of weakness nor incompetence to seek the advice or assistance of a more experienced colleague in such cases. There is a balance between accepting a challenging case over which you have a high degree of control, even if the outcome may be less predictable, and foolishly taking on a case either for financial reward or because of undue pressures placed on you by the patient or other parties. These parties could be an employer, colleague, payment agency or commissioning body. ${ }^{16}$

Working within some systems, like the NHS, may make certain treatments and procedures appear to be financially unviable but it would be considered unethical and unprofessional to refer a patient simply on this basis alone. Indeed the
NHS contract is explicit in this: 'In making a decision as to what services to recommend or provide to a patient who has sought services under the contract; or to refer a patient for other services by another contractor, hospital or other relevant service provider, the contractor shall do so without regards to its own financial interests. ${ }^{17}$

When a referral is made it is important to ensure a system exists in the practice to track it, particularly those that are urgent or high risk by virtue of their need, eg suspected malignancy. The specialist may not receive the referral either because it was not made, not received or lost in various systems. The practice should follow up referrals to make sure they are dealt with and this can be supplemented by copying the referral letter to the patient or telephoning the patient after a certain period to make sure that an appointment has been made and kept. It might be appropriate also to empower the patient to contact the practice if nothing is heard after say 7-10 days in the case of urgent referrals.

\section{RISK MANAGEMENT}

All this has been a preamble to what is known as risk management. The giving of appropriate advice and warnings, the completion of accurate records and appropriate referrals are the cornerstones of recognising problems before they happen and dealing with them when they do.

Risk management in dentistry has been developed over the years by concentrating on recording treatment in dental records and informing patients of the proposed treatment before treating them. ${ }^{18}$ Conversely, failure in communication has been identified as the predominant factor in $80 \%$ of patient complaints and litigation.

Risk management covers all the processes involved in identifying, assessing and judging risks, taking actions to mitigate or anticipate them, and monitoring and reviewing progress. Risk is a factor to be considered during any clinical care and the successful outcome of treatment leading to patient satisfaction requires risks to be contained and controlled.

Risk can be defined as uncertainty of outcome (whether positive opportunity or negative threat). Most interventive clinical procedures come with some degree of risk but the aim of risk management is to manage the patient's exposure to risk - ie the probability of specific risks occurring and the potential impact if they did occur.

The management of risk is not a linear process; rather it is the balancing of a number of interwoven elements which interact with each other and which have to be in balance with each other. The classic risk management cycle proposes the following four stages:

1. Risk identification - what can go wrong?

2. Risk analysis - what are the chances of it going wrong and what are the consequences?

3. Risk control - what can be done to control the risk?

4. Risk transfer - should you assume the risks or transfer them, for example by referring the patient?

Equally important in risk management is learning from mistakes, adverse incidents or near misses. A near miss is an occurrence which but for luck or skilful management ${ }^{19}$ would have become an incident. Using root cause analysis tools such as the five why technique and the Ishikawa cause and effect 'fishbone' diagram gives answers to what happened, why it happened and what can be done to prevent it happening again.

There is much to be learnt both by the practitioner who makes the error and the practice they work in, since, when analysed carefully, the error will have arisen as much from human error as system based errors where the pre-existing conditions or environmental factors pertaining at the time make the error more likely. At its most basic, for example if there is no system to update medical histories, postoperative extraction complications may occur if there was a failure to recognise the patient was on a high dose of anticoagulants. The human error was failing to ask the patient (active failure) but the system failure was that there was no routine process (latent failure) for collecting this information..$^{20}$

The mark of a professional is to acknowledge when things go wrong, communicate this to patients with humility and to learn from the problem to ensure that, where possible, it does not occur again. 
1. Bunting R F Jr, Benton J, Margan W D. Practical risk management for physicians. J Healthc Risk Manag 1998; 18(4): 29-53.

2. Rattan $\mathrm{R}$, Tiernan J. Risk management in general dental practice. London: Quintessence Publishing, 2004.

3. Bailey D. Recovery from customer service shortfalls. Managing Service Quality 1994; 4(6): 25-28.

4. DiMatteo M R, Hays R D, Prince L M. Relationship of physicians' non-verbal communication skills to patient satisfaction, appointment noncompliance, and physician workload. Health Psychol 1986; 5: 581-594.

5. Anon. Influencing satisfaction. In Annual review 2006. What the patient did next. pp 6. London: Dental Protection Ltd, 2006

6. Dental Protection Ltd. Dental news 21 - risk management advice. London: Dental Protection Ltd, 2000.

7. D'Cruz L. Who cares for the carers? Br Dent J 2009; 207: 11-12.
8. D'Cruz L. Off the record. Dent Update 2006; 33: 390-400.

9. Watt $R, M c G l o n e ~ P, E v a n s ~ D$ et al. The facilitating factors and barriers influencing change in dental practice in a sample of English general dental practitioners. Br Dent J 2004: 197: 485-489.

10. Faculty of General Dental Practice (UK). Clinical examination and record-keeping: good practice guidelines. 2nd ed. London: Faculty of General Dental Practice (UK), 2009.

11. Beaglehole R H, Watt R G. Helping smokers stop. A guide for the dental team. London: Health Development Agency, 2004.

12. National Radiological Protection Board. Guidance notes for dental practitioners on the safe use of $X$-ray equipment. Didcot: National Radiological Protection Board, 2001.

13. Dental Protection Ltd. No way back? The perils of elective treatment. Riskwise UK 2002; 24: 9-10.

14. General Dental Council. Standards for dental professionals. Paragraph 1.3. London: General Dental Council, 2005

15. Dental Protection Ltd. Over to you - changes in the UK referral culture. Riskwise UK 2006; 31: 8.

16. Dental Protection Ltd. Competence. Dental Ethics Module 10. London: Dental Protection Ltd, 2007. ISSN 1752-6809.

17. Department of Health. Standard general dental services contract. Part 15, paragraph 243. London: Department of Health, 2009.

18. Graskemper J P. A new perspective on dental malpractice: practice enhancement through risk management. J Am Dent Assoc 2002; 133: 752-757.

19. Lugon M, Secker-Walker J. Clinical governance: making it happen. pp 77-92. London: Royal Society of Medicine Press, 1999.

20. Department of Health. An organisation with a memory. Report of an expert group on learning from adverse events in the NHS chaired by the Chief Medical Officer. London: Department of Health, 2000. 